\section{G213(P) END-OF-LIFE CARE DECISIONS: DIFFERING DECISION MAKING PROCESSES}

E Popejoy. School of Health Sciences, University of Nottingham, Nottingham, UK

\subsection{6/archdischild-2015-308599.207}

Aim Parents of children with life-limiting illnesses (LLI) are often required to make decisions regarding what type of life-sustaining treatment, if any, is to be provided and where care is to be delivered at the end of their child's life. This study aimed to explore the experiences of parents in making and revising endof-life care decisions for their child with a LLI.

Methods Semi-structured interviews were conducted with three bereaved parents of children with LLI between April and July 2014. Data were analysed using Interpretative Phenomenological Analysis. Eligible families were invited to participate by the child's main consultant. Approval for the study was granted from the National Research Ethics Service and written informed consent was obtained prior to the interview.

Results Two parents in the study had received their child's diagnosis at least a year prior to their child's death, whereas the other parent had only received the diagnosis in the final weeks of the child's life. This parent, prior to receiving the diagnosis, had made decisions to treat her child aggressively on the presumption that the illness was curable. Upon receiving the diagnosis, she decided in conjunction with professionals to transition towards palliative care and ensuring comfort for her child. The other parents had been aware of their child's diagnoses for longer and deemed from the start that aggressive treatment would not be appropriate or in their child's best interests.

Conclusion The majority of the literature on the topic of decision making in children's palliative care includes children with both life-limiting and life-threatening illnesses (LTI). Although there may be some overlap in the decision making processes, this study although limited by its sample, has shown that decisions may differ for children with LLI as compared to children with LTI, suggesting that perhaps these populations should be researched separately. More research is needed in order to determine the differences in the decision making processes and whether specific support with decision making is needed between the two groups.

\section{G214(P) BLENDED FOOD FOR ENTERAL FEEDING VIA A GASTROSTOMY}

SJ Brown. Care Team, Children's Hospice Southwest, Barnstaple, UK

10.1136/archdischild-2015-308599.208

A blended diet for enteral nutrition is defined as home-made everyday food blended to a smooth "single cream" consistency. Currently blended food is not recommended by the British Dietetic Association as a first choice supporting guidance on enteral feeding from N. I. C. E. However in a UK children's hospice the policy is where possible to replicate the care received at home, respecting parents' wishes. Therefore guidelines needed to be created for its use in the hospice. Benefits in physical and emotional health needed to be balanced against risks in tube blockage, contamination and digestive upsets.

Aim was to gather information and risk assess the processes in order to formulate guidelines on the use of blended food for staff and parents.
The process involved searches for information on the internet and liaising with specialist groups of dieticians and tube manufacturers. For the process of syringing/plunging where there is very little information available a visual demonstration was developed using a plastic box and a gastrostomy button. For issues relating to contamination and food hygenine we used exsisting guidance on food preparation.

Guidelines were developed and presented to our clinical governance group for consideration and acceptance for use.

Working with local dieticians a package of information including all the considerations and risks where developed to give to parents.

Blended food is not recommended as a primary choice for enteral feed and may not be supported by all those involved in a childs care such as schools or respite unit. Blended diet is used by $10 \%$ of the tube fed children using our service. With careful consideration we have been able to develop practical guidelines that support and replicate care given at home whilst respecting the choices made by all our families on how their children are feed.

For the future one UK university has started research to look at the health, social benefits and implications of blended diet. In North America where there are prescription costs and strict insurance schemes many opt for blended food, for others this is simply their preferred method. Here a company has developed a shelf safe product for gastrostomy feeding from "real food" available on Amazon.com

\section{G215 SETTING AND MAINTAINING PROFESSIONAL BOUNDARIES IN PAEDITRIC CLINICAL PERSPECTIVES FROM AN INTERPROFESSIONAL PERSPECTIVE}

${ }^{1} \mathrm{R}$ Bolland, ${ }^{2} \mathrm{~L}$ Roderique. ${ }^{1}$ Paeditric Unit, St George's Healthcare NHS Trust, London, UK; ${ }^{2}$ Faculty of Health, Social Care and Education, Kingston University, London, UK

10.1136/archdischild-2015-308599.209

Aim This study explored the manner in which paediatric nurses and hospital play specialists set professional boundaries and the challenges they faced in maintaining a therapeutic relationship when caring for children and their families in hospital.

Methods A phenomenological approach was adopted, through a purposive sample of seven paediatric settings within one NHS Trust. Eight paediatric nurses and two hospital play specialists were interviewed. Thematic analysis, using field notes and transcribed tape recorded interviews, was used to identify emerging themes and generate a description of the respondents' experience.

Results The study revealed that paediatric nurses had difficulty in defining the concept of professional boundaries, therapeutic relationships and self disclosure. Although no clear definition was given for professional boundaries, respondents agreed that they were important. Paediatric nurses could recognise when boundaries were being broken but lacked the confidence to address boundary violations. Using self disclosure was seen as a balancing act which could lead to boundary crossing and boundary violation. Nurses felt unsupported by senior colleagues particularly when faced with "manipulative families" and found their code of conduct ${ }^{1}$ helped guide their practice. Hospital play specialists had a greater understanding of therapeutic relationships then paediatric nurses.

Conclusions Health care professionals can recognise professional boundaries and boundary violations but they need support to 